\title{
Optimizing control flow in loops using interval and dependence analysis
}

\author{
Mohammad Ali Ghodrat • Tony Givargis • Alex Nicolau
}

Received: 19 January 2009 / Accepted: 16 June 2009 / Published online: 1 July 2009

(C) The Author(s) 2009. This article is published with open access at Springerlink.com

\begin{abstract}
We present a novel loop transformation technique, particularly well suited for optimizing embedded compilers, where an increase in compilation time is acceptable in exchange for significant performance increase. The transformation technique optimizes loops containing nested conditional blocks. Specifically, the transformation takes advantage of the fact that the Boolean value of the conditional expression, determining the true/false paths, can be statically analyzed using a novel interval analysis technique that can evaluate conditional expressions in the general polynomial form. Results from interval analysis combined with loop dependency information is used to partition the iteration space of the nested loop. In such cases, the loop nest is decomposed such as to eliminate the conditional test, thus substantially reducing the execution time. Our technique completely eliminates the conditional from the loops (unlike previous techniques) thus further facilitating the application of other optimizations and improving the overall speedup. Applying the proposed transformation technique on loop kernels taken from Mediabench, SPEC-2000, mpeg4, qsdpcm and gimp, on average we measured a $2.34 \mathrm{X}$ speedup when running on a UltraSPARC processor, a 2.92X speedup when running on an Intel Core Duo processor, a 2.44X speedup when running on a PowerPC G5 processor and a 2.04X speedup when running on an ARM9 processor. Performance improvement, taking the entire application into account, was also promising: for 3 selected applications (mpeg-enc, mpeg-dec and qsdpcm) we measured 15\% speedup on best case (5\% on average) for the whole application.
\end{abstract}

Keywords Interval analysis · Compiler loop optimization · Algorithmic code transformation · Control flow optimization

M.A. Ghodrat ( $\varangle) \cdot$ T. Givargis · A. Nicolau

Department of Computer Science, University of California, Irvine, CA, USA

e-mail:mghodrat@uci.edu

T. Givargis

e-mail: givargis@uci.edu

A. Nicolau

e-mail: nicolau@uci.edu 


\section{Introduction}

Software is becoming a larger fraction of engineering effort. Aggressive compiler optimization, in particular those that address loops can significantly improve the performance of the software, thus justifying the additional compilation time requirements. This is in particular true in the embedded system domain where software has become a key element of the design process and performance is of a critical concern. Furthermore, it is acceptable for a compiler intended for embedded computing to take longer to compile but perform aggressive optimizations, such as the ones presented in [16]. In our case, the additional compiler execution time was of the order of 10 mili seconds per loop [3].

In contrast to existing work on loop transformation, we present an algorithmic loop transformation technique that substantially restructures the loop using knowledge about the control flow combined with data-dependence information within the body of the loop. The control flow and data-dependences within the loop body are analyzed using a static interval analysis technique previously outlined in [3]. Interval analysis provides information on the true/false paths within the original loop body as a function of the loop indices. The analysis of the loop iteration dependencies is used to establish the possible space of loop restructuring. Combining these two static analysis results, an algorithm is provided that fully partitions the original iteration space (i.e., original loop) into multiple disjoint iteration spaces (i.e., generated loops). The bodies of these generated loops are void of conditional branches and thus (unlike previous techniques which leave branches in loops) our techniques allows for more effective optimizations. Moreover, each of these loops, and the ordering within them, are consistent with the original loop iteration dependencies.

As an example consider the loop kernel shown below. This loop kernel is taken from gimp benchmark [14].

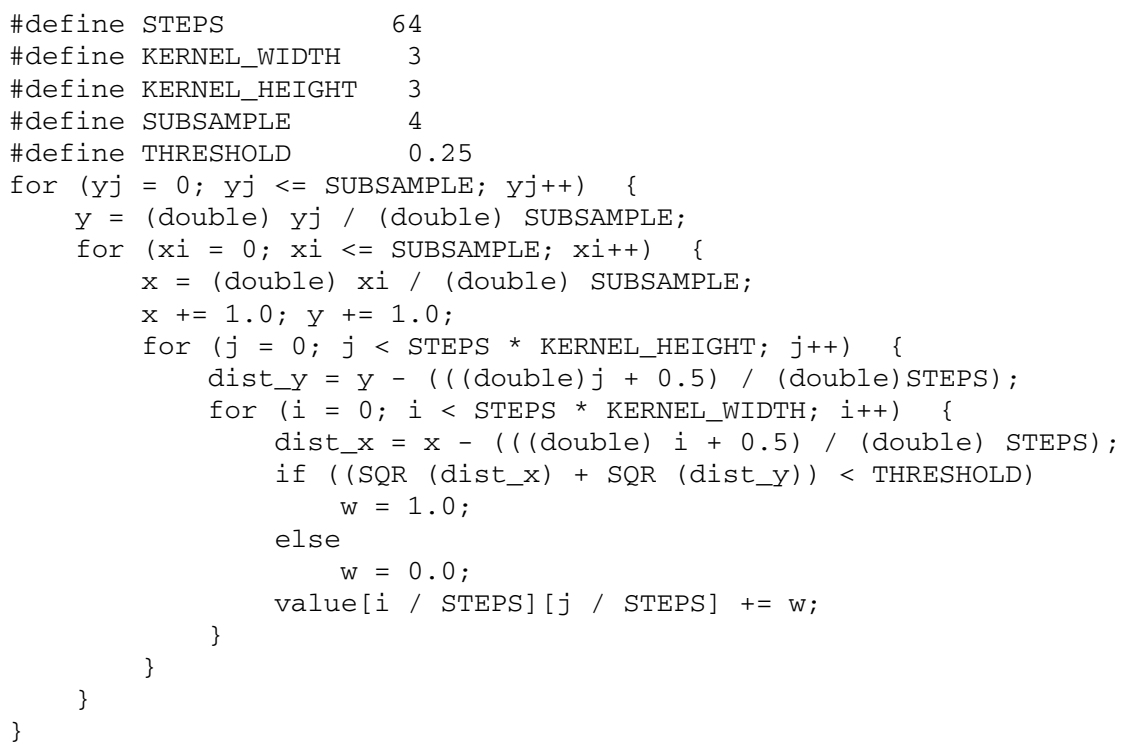

Using interval analysis [3] we statically compute information as shown in the table of Fig. 1 on the conditional expression in the loop nest $\left(\left(S Q R\left(\right.\right.\right.$ dist $\left._{x}\right)+S Q R\left(\right.$ dist $\left.\left.\left._{y}\right)\right)<T H R E S H O L D\right)$. For example the 2 nd row of this table shows that when $(0 \leq x i \leq 1) \& \&(0 \leq y j \leq 1)$ $\& \&(152 \leq i \leq 191) \& \&(0 \leq j \leq 191)$ the expression $\left(\operatorname{SQR}\left(\operatorname{dist}_{x}\right)+\operatorname{SQR}\left(\right.\right.$ dist $\left.\left._{y}\right)\right)<$ 
Fig. 1 Interval analysis result for the expression $\left(S Q R\left(\right.\right.$ dist $\left._{x}\right)+$ $\operatorname{SQR}\left(\right.$ dist $\left.\left._{y}\right)\right)<$ THRESHOLD

\begin{tabular}{ll}
\hline Space $([\mathrm{xi}][\mathrm{yj}][\mathrm{i}][\mathrm{j}])$ & Evaluation result(false/true) \\
\hline$[0,1][0,1][152,191][0,191]$ & false \\
{$[0,1][2,2][123,191][0,191]$} & false \\
{$[0,1][3,4][157,191][0,191]$} & false \\
{$[2,2][0,1][0,28][0,191]$} & false \\
{$[2,2][0,1][163,191][0,191]$} & false \\
{$[2,2][2,2][0,63][0,191]$} & false \\
{$[2,2][2,2][128,191][0,191]$} & false \\
{$[3,4][0,1][0,40][0,191]$} & false \\
{$[3,4][2,2][0,68][0,191]$} & false \\
{$[3,4][3,4][0,34][0,191]$} & false \\
\hline
\end{tabular}

THRESHOLD evaluates to false. The transformed code, using the 2 nd row of table yields the optimized code shown below:

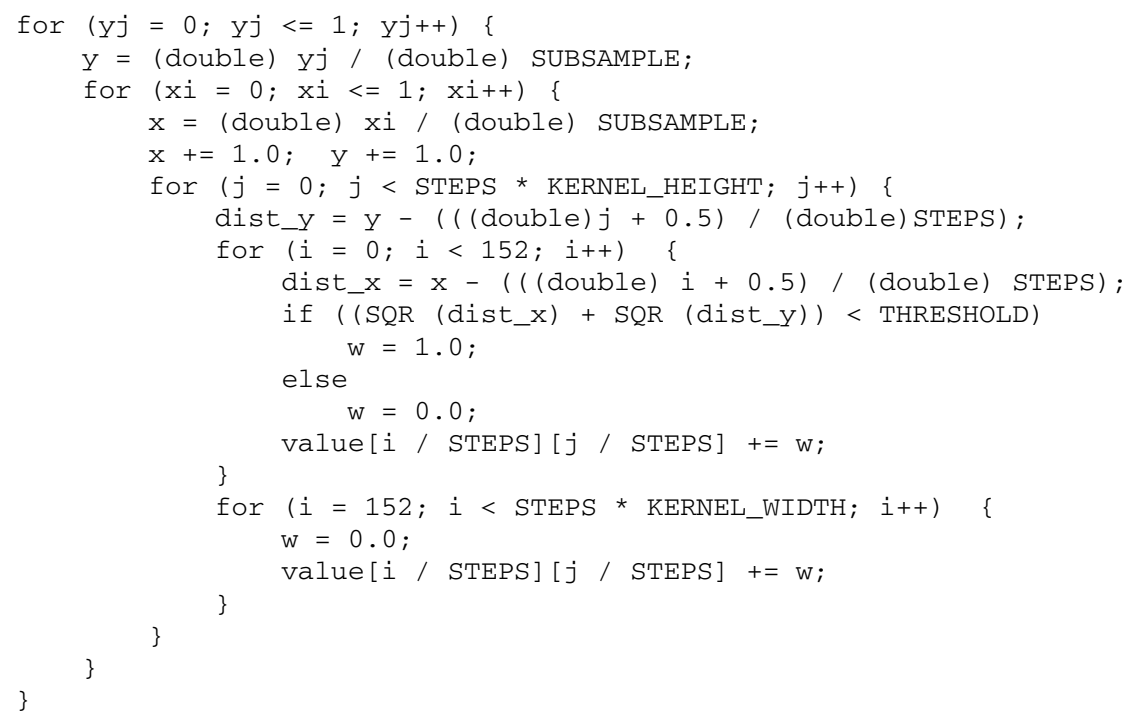

In the transformed code, the evaluation of the conditional expression for part of the most inner loop (i.e., the loop with $i$ as the index variable) is eliminated. Applying our optimization to the rest of the loop kernel, while using the entire information in Table 1, we obtain $16 \%$ speed-up on SPARC, $21 \%$ on Intel Core Duo and 24\% on PowerPC G5 as shown in Sect. 5.

The rest of this paper is organized as follows. In Sect. 2, we outline the related work. In Sect. 3, we formulate the problem, show the overall flow of the proposed transformation and establish some preliminaries. In Sect. 4, we establish the transformation technique. In Sect. 5, we show our experimental results. In Sect. 6, we conclude.

\section{Previous work}

There are many transformation techniques targeting nested loops. Since our work specifically applies to control flow optimization of loops we primarily focus on related work that target control flow optimization. Of course, data-flow level optimizations can be combined 
Table 1 Properties which are being compared in Table 2

\begin{tabular}{|c|c|}
\hline Property 1 & $\begin{array}{l}\text { Optimize control flow of a loop with nested conditional } \\
\text { block }\end{array}$ \\
\hline Property 2 & Dependence analysis needed \\
\hline Property 3 & Conditional expression depends on loop index \\
\hline Property 4 & $\begin{array}{l}\text { Conditional expression is an affine function of loop } \\
\text { variables }\end{array}$ \\
\hline Property 5 & Conditional expression contains logical operators \\
\hline Property 6 & $\begin{array}{l}\text { Conditional expression is a function of loop indices and } \\
\text { non-loop-index variables }\end{array}$ \\
\hline Property 7 & Conditional expression has a general polynomial form \\
\hline Property 8 & $\begin{array}{l}\text { Conditional expression will be removed completely } \\
\text { from loop body of the transformed code }\end{array}$ \\
\hline
\end{tabular}

with control flow optimizations to further improve the generated code (i.e., data-flow optimizations may benefit from simpler control flow within loops).

Table 1 provides a set of properties that are used to compare and contrast loop optimization strategies using control flow analysis. Furthermore, Table 2 summarizes existing loop transformation techniques and provides an analysis of their strength relative to the presented work.

Among all the techniques listed in Table 2, the three most relevant ones are loop unswitching, index-set splitting and loop nest splitting.

Loop unswitching [9], has similarities to our transformation in targeting conditional blocks within loops. Specifically, loop unswitching attempts to replicate the loop inside each branch of the conditional. In contrast, our technique attempts to completely eliminate the conditional block within a loop by decomposing a loop into multiple independent loops. In loop unswitching technique, the conditional expression does not depend on loop indices, hence limiting its applicability to loops containing trivial conditions, but in our technique the conditional expression is a function of loop indices.

Another technique, index-set splitting [15], does a similar transformation but in a much limited way than our method. First index-set splitting only considers affine expressions and there is no discussion on how to handle cases where there are dependences between loop iterations. In our method we consider non-affine conditional expressions within the loop and handle cases where there are dependences between loop iterations and, when dependences allow, we eliminate the conditionals from the loops.

A closely related work in control flow loop optimization is suggested by Falk et al. [2]. The loop model used in their work differs from ours. First, they consider conditional expressions that are strictly affine (vs. arbitrary polynomial in our case) functions of the loop indices. Figure 2a shows a case in gimp [14] benchmark which is optimized by our technique but not by their method. Second, Falk's loop model assumes that the conditional expression is strictly a function of loop indices, but in our loop model the conditional expression can include other variables computed within the loop body. Figure $2 \mathrm{~b}$ shows a case in $m p 3$ benchmark [1] that can be optimized by our technique but not by their method (here the transformed code is not shown to save space). The final important difference between our work and Falk's is that in our transformed code the conditional block is completely eliminated while in their work it is simplified or hoisted to a higher point in the nested loops, but not eliminated. To show this difference clearly, let's first consider a synthetic example shown in Fig. 2c. Figure 2c shows a case in which our technique (Fig. 2e) has remove the condition completely resulting in significant (30\% on SPARC and $68 \%$ on Intel) speedup while their technique (Fig. 2d) has only partially eliminated the evaluation of the conditional 
Table 2 Comparison with other loop optimization techniques

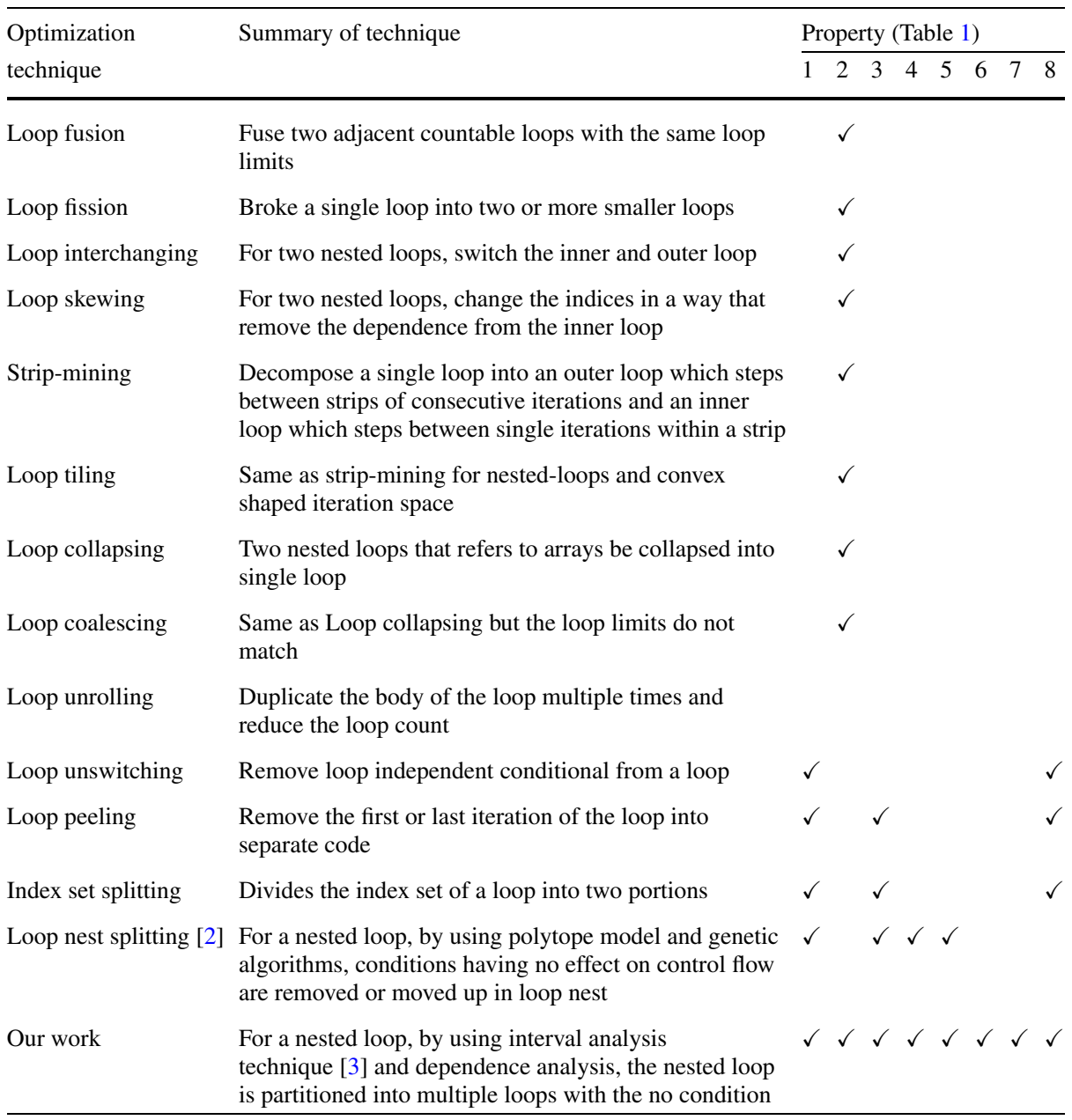

expression. A similar example 186.crafty from SPEC-2000 [10] is shown in Fig. 2f where applying the technique in [2] will not remove the conditions completely.

\section{Proposed transformation}

The proposed transformation decomposes the original nested loops of Fig. 3a into three parts, as shown in Fig. 3b. The first part sets up one or more nested loop structures with iteration spaces for which the $s t_{\text {cond_expr }}$ is known to be true at compile time. Likewise, the second part sets up one or more nested loop structures with iteration spaces for which the $s t_{\text {cond_expr }}$ is known to be false at compile time. The third part sets up one or more nested loop structures with an iteration space for which the $s t_{\text {cond_expr }}$ can not be statically evaluated. The three parts combined cover the entire iteration space of the original nested loops. Since the evaluation of $s t_{\text {cond_expr }}$ is eliminated in parts one and two, the decomposed code executes substantially fewer instructions than the original code. 


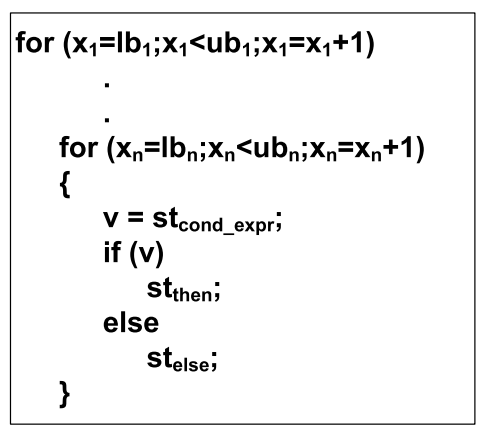

(a)

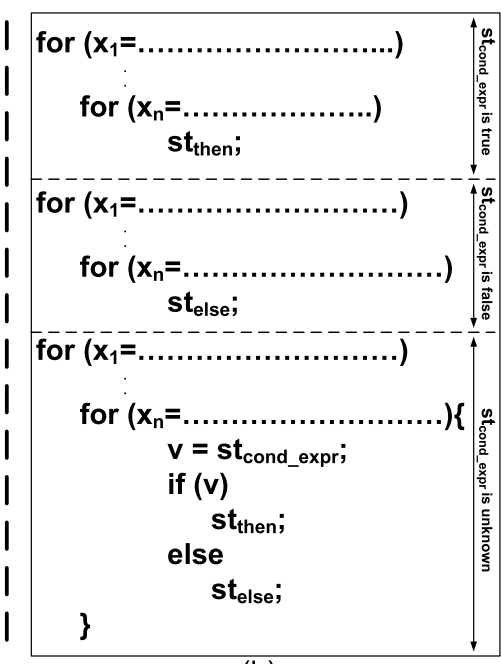

(b)

Fig. 3 Transformation

plex condition is either a simple condition or two complex conditions merged using logical operators $(\& \&, \|, !)$.

An integer interval of the form $[a, b]$ represents all possible integer values in the range $a$ to $b$, inclusively. The operations $(+,-, \times, /)$ can be defined on two intervals $[a, b]$ and $[c, d]$. We refer the interested reader to [8] for a full coverage of interval arithmetic.

We define an $n$-dimensional space to be a box-shaped region defined by the Cartesian product $\left[l_{0}, u_{0}\right] \times\left[l_{1}, u_{1}\right] \times \cdots \times\left[l_{n-1}, u_{n-1}\right]$. Hence, for a given program with $n$ input integer-variables $x_{0}, x_{1}, \ldots, x_{n-1}$, the program domain space is an $n$-dimensional space defined by the Cartesian product $\left[\min _{0}, \max _{0}\right] \times\left[\min _{1}, \max _{1}\right] \times \cdots \times\left[\min _{n}, \max _{n}\right]$, where $\min _{i}$ and $\max _{i}$ are defined based on the type of the variable $x_{i}$ (e.g. if $x_{i}$ is of type signed character then $\min _{i}=-128$ and $\max _{i}=127$ ).

\section{Technical approach}

We now begin to describe the technique proposed in this paper. A candidate loop $L$ has the structure shown in Fig. 3a. The iteration space of $L$ is defined as $\left[l b_{1}, u b_{1}\right] \times\left[l b_{2}, u b_{2}\right]$ $\times \cdots \times\left[l b_{n}, u b_{n}\right]$. The body of $L$ can be decomposed into the reducible CDFGs corresponding to $s t_{\text {cond_expr }}$, st then, and $s t_{\text {else }}$. The variable $v$, computed by $s t_{\text {cond_expr }}$, is defined in terms of the loop variables $x_{1}, x_{2}, \ldots, x_{n}$ and all other variables which are alive when computing the value of $v$. The transformation technique consists of a number of steps, specifically:

- Compute the interval set of $v$ by processing the CDFG corresponding to $s t_{\text {cond_expr }}$ (Sect. 4.1).

- Compute the dependence vector of iteration space (Sect. 4.2).

- Partition the iteration space (Sect. 4.3).

- Generate code (Sect. 4.4).

Given the conditional expression cond_expr with variables $x_{1}, x_{2}, \ldots, x_{k}$, the domain space partitioning problem [3] is to partition the domain space of cond_expr into a mini- 
Fig. 4 Partitioned domain of $2 x_{0}+x_{1}+4>0$

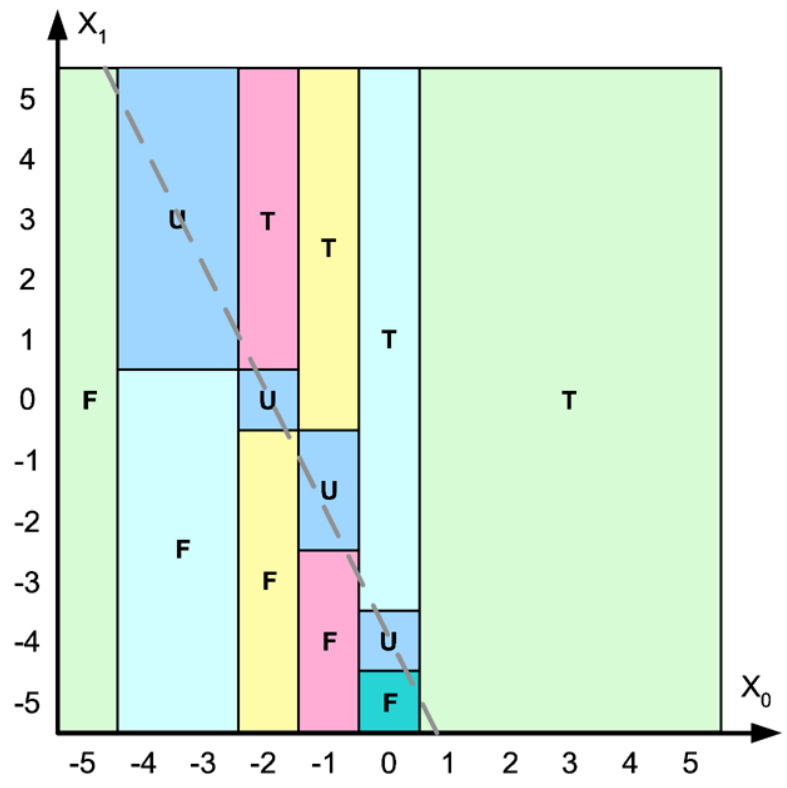

mal set of $k$-dimensional spaces $s_{1}, s_{2}, \ldots, s_{n}$ with each space $s_{i}$ having one of true(T), false(F), or unknown(U) Boolean value. If space $s_{i}$ has a Boolean value of true, then cond_expr evaluates to true for every point in space $s_{i}$. If space $s_{i}$ has a Boolean value of false, then cond_expr evaluates to false for every point in space $s_{i}$. If space $s_{i}$ has a Boolean value of unknown, then cond_expr may evaluate to true for some points in space $s_{i}$ and false for others.

For example, consider cond_expr: $2 \times x_{0}+x_{1}+4>0$ (domain space $\left.[-5,5] \times[-5,5]\right)$. Figure 4 shows the partitioned domain space and the corresponding Boolean values [3].

\subsection{Interval set computation}

In the following discussion, the code segment presented in Table 3 is used to demonstrate the interval_set computation. In Table 3, loop variables $x_{1}$ and $x_{2}$ are assumed to be live on entry (i.e., inputs to the $s t_{\text {cond_expr }}$ CDFG) and Boolean variable $v$ is assumed to be live on exit (i.e., output of the $s t_{\text {cond_expr }}$ CDFG). We refer the reader to Sect. 3.1 for a review of integer intervals, spaces and program domain space used here.

At any given point in the CDFG, a variable $v$ has an interval, defining the range of possible values it may have. At the point of declaration, the type of a variable $v$ gives the upper and lower bounds of such an interval (e.g., line 1 of Table 3). Along each path in the CDFG, originating from the point of declaration of $v$, we recompute $v$ 's interval when $v$ is redefined according to the following rules:

- If $v$ is assigned a constant value $C$ (or, expression evaluating to a constant value), then $v$ 's interval is defined to be $[C, C]$.

- If $v$ is assigned a unary arithmetic expression in the form of $v=O P x_{i}$, then $v$ 's interval is defined to be the corresponding arithmetic operation $O P$ applied to $x_{i}$ 's interval.

- If $v$ is assigned a binary arithmetic expression in the form of $v=x_{i} O P x_{j}$, then $v$ 's interval is defined to be the corresponding arithmetic operation $O P$ applied to $x_{i}$ 's and $x_{j}$ 's intervals. 
Table 3 Interval-set example

\begin{tabular}{llll}
\hline Code $\left(s t_{\text {cond_expr }}\right)$ & Interval & Condition & Space \\
\hline$/ /$ loop var: $x_{1}$ & {$[-10,10]$} & & \\
// loop var: $x_{2}$ & {$[-5,5]$} & & \\
1: bool $v ;$ & {$[0,1]$} & true & {$[-10,10] \times[-5,5]$} \\
2: $v=0 ;$ & {$[0,0]$} & true & {$[-10,10] \times[-5,5]$} \\
3: if $\left(x_{1}>0 \& \& x_{2}>0\right)$ & & & \\
$4: v=1 ;$ & {$[1,1]$} & $\left(x_{1}>0 \& \&\right.$ & {$[1,10] \times[1,5]$} \\
& & $\left.x_{2}>0\right)$ & \\
\hline
\end{tabular}

- If $v$ is assigned a complex arithmetic expression, then the complex arithmetic expression is decomposed into a set of unary or binary operations as defined above.

- If $v$ is assigned a statically undeterminable function, than $v$ 's interval is defined according to its type.

Let us extend the notion of $v$ 's interval by associating a conditional expression with $v$ 's interval (third column in Table 3). The goal is to capture the fact that $v$ 's interval takes on different values along different paths (forks based on conditional expression) in the CDFG. For example, line 4 of Table 3 shows a conditional assignments to variable $v$, based on the values of the input variables $x_{1}$ and $x_{2}$. In this example, when $\left(x_{1}>0\right) \& \&\left(x_{2}>0\right) v$ 's interval is defined to be $[1,1]$, otherwise, $v$ 's interval is defined to be $[0,0]$.

Let us establish an equivalence between a conditional expression and a set of spaces (fourth column in Table 3). For each conditional expression cond_expr, there exists a set of spaces $S_{1}, S_{2}, \ldots, S_{k}$ that collectively defines the part of the domain space for which cond_expr evaluates to true. For example, line 4 of Table 3 shows the conditional expression $\left(x_{1}>0\right) \& \&\left(x_{2}>0\right)$ defined as $[1,10] \times[1,5]$.

Formally, for a variable $v$, the interval_set (i.e., v.iset) is defined as $\left\{\left(I_{j}, S_{j}\right) \mid j \in\right.$ $(1 \ldots m)\}$, where $I_{j}$ is an integer interval and $S_{j}$ a space. Furthermore, $\bigcup_{j=1}^{m} S_{j}=$ iteration_space. Intuitively, the interval_set captures the range of values that a variable may receive during the execution of a program, taking the control flow into account.

A procedure for computing the output interval-set of a reducible CDFG follows:

(1) Topologically sort the CDFG's basic blocks and obtain $b_{0}, b_{1}, \ldots, b_{n}$, repeat steps 2-5 for each basic block in sorted order.

(2) Compute the interval set(s) for every DFG in $b_{i}$.

(3) Perform domain space partitioning analysis on the conditional expression at the exit of $b_{i}[3]$.

(4) Use the true and unknown spaces to compute the interval set(s) of the input variables of $b_{i}$ 's jump-through basic block.

(5) Use the false and unknown spaces to compute the interval set(s) of the input variables of $b_{i}$ 's fall-through basic block.

Applying the above algorithm on the $s t_{\text {cond_expr }}$ CDFG would yield the interval_set of the Boolean variable $v$ :

$$
\begin{aligned}
\text { v.iset }= & \left\{\left([1,1], S_{T 1}\right),\left([1,1], S_{T 2}\right), \ldots,\left([1,1], S_{T n_{1}}\right),\right. \\
& \left([0,0], S_{F 1}\right),\left([0,0], S_{F 2}\right), \ldots,\left([0,0], S_{F n_{2}}\right), \\
& \left.\left([0,1], S_{U 1}\right),\left([0,1], S_{U 2}\right), \ldots,\left([0,1], S_{U n_{3}}\right)\right\} .
\end{aligned}
$$




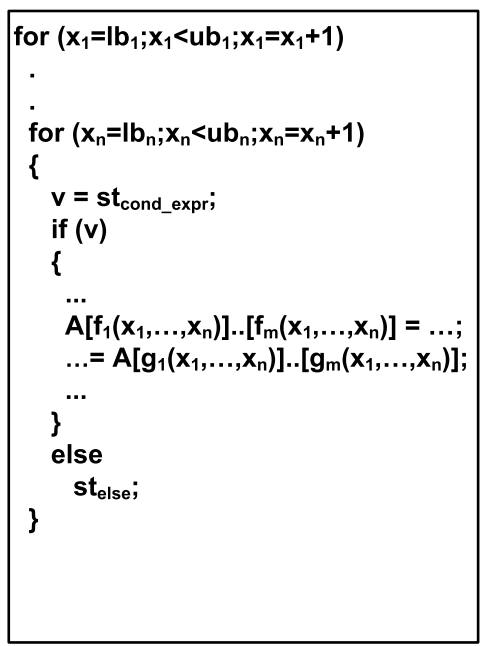

(a)

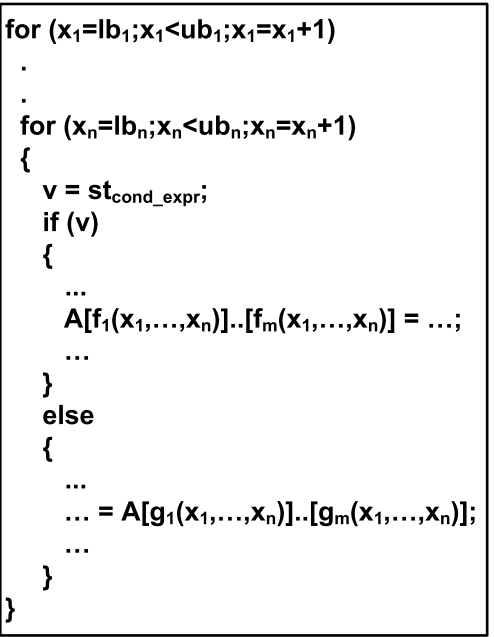

(b)

Fig. 5 General memory access model

Furthermore, we define three sets of spaces:

$$
T=\left\{S_{T 1}, S_{T 2}, \ldots, S_{T n_{1}}\right\}, \quad F=\left\{S_{F 1}, S_{F 2}, \ldots, S_{F n_{2}}\right\}, \quad U=\left\{S_{U 1}, S_{U 2}, \ldots, S_{U n_{3}}\right\} .
$$

For the example of Table 3, the interval_set of the Boolean variable $v$ is:

$$
\text { v.iset }=\{([1,1],[1,10] \times[1,5]),([0,0],[-10,0] \times[-5,5]),([0,0],[1,10] \times[-5,0])\} \text {. }
$$

\subsection{Dependence vector computation}

Data dependency in a loop is either of type loop-carried or of type loop-independent. Loopindependent dependency occurs when at least one of the statements $s t_{1}$ and $s t_{2}$ write the memory location $M$ during the same loop iteration. Loop-carried dependency occurs when statement $s t_{1}$ accesses the memory location $M$ in one iteration and $s t_{2}$ accesses it in some iteration later and at least one of these accesses is a write. In this discussion, statements $s t_{1}$ and $s t_{2}$ may belong to any of $s t_{\text {cond_expr }}, s t_{\text {then }}$ or $s t_{\text {else }}$.

For each iteration of the nested loop structure, we define a vector $I=\left\{i_{1}, \ldots, i_{n}\right\}$ of integers showing the corresponding values of the loop indices. If there is a data dependency between statement $s t_{1}$ during iteration $I=\left\{i_{1}, \ldots, i_{n}\right\}$ and statement $s t_{2}$ during iteration $J=\left\{j_{1}, \ldots, j_{n}\right\}$, then the dependence vector is defined as $J-I=\left\{j_{1}-i_{1}, \ldots, j_{n}-i_{n}\right\}$.

The notion of dependence vector is well established in the compiler literature [6]. The existing dependence vector analysis techniques make the conservative assumption that any pair of statements within a loop body may execute during the same iteration. For the proposed transformation, we extend the analysis of dependence vector to account for control flow dependency between a pair of statements with the loop body, as described below.

Figure 5 shows our general $m$-dimensional memory access model. Figure 5 a shows the case when both statements access an array during the execution of the then part. Figure $5 \mathrm{~b}$ shows the case when one statement accesses an array during the execution of the then part and the other statement accesses an array during the execution of the else part. 


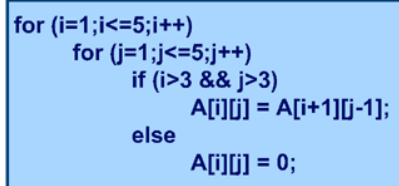

(a)

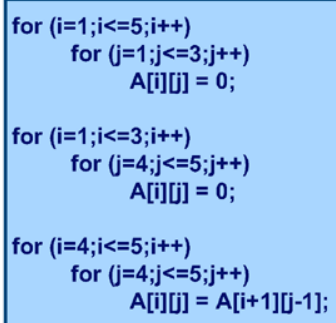

(b)

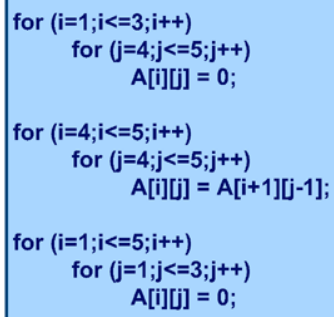

(c)

Fig. 6 (a) Original code; (b) Wrong transformed code; (c) Correct transformed code

In the case of Fig. 5a, there exists a data dependence if there are two iteration vectors $I$ and $J$ such that:

$$
\begin{aligned}
& f_{k}(I)=g_{k}(J) \forall k, 1 \leq k \leq m \& \& \\
& s t_{\text {cond_expr }}(I)=\text { true \&\& st cond_expr }(J)=\text { true }
\end{aligned}
$$

In the case of Fig. 5b, there exists a data dependence if there are two iteration vectors $I$ and $J$ such that:

$$
\begin{aligned}
& f_{k}(I)=g_{k}(J) \forall k, 1 \leq k \leq m \& \& \\
& s t_{\text {cond_expr }}(I)=\text { true \&\&st } t_{\text {cond_expr }}(J)=\text { false }
\end{aligned}
$$

In the case that both of the accesses are in the else part, then $s t_{\text {cond_expr }}(I)$ and $s t_{\text {cond_expr }}(J)$ in (1) are equal to false. Similarly, the case when the write access is in the else part and the read access is in the then part, $\operatorname{st}_{\text {cond_expr }}(I)=$ false and $s t_{\text {cond_expr }}(J)=$ true in (2).

\subsubsection{Example}

Before going over the next step of our methodology, an example will be presented which shows the importance of the dependence vector computation and how it effects the result of iteration space partitioning if it is ignored. Intuitively, what we need to ensure is that the order of dependent statements from different spaces is preserved by the execution order of the transformed loops. That is, a space (loop) $S_{i}$ has to execute before space (loop) $S_{j}$ if there are dependences between statements in $S_{i}$ and statements in $S_{j}$ - e.g., if a statement in $S_{i}$ produces a values used in $S_{j}$. As an example, consider the simple code segment shown in Fig. 6a.

The result of domain space partitioning for the expression $(i>3 \& \& j>3)$ is given in Fig. 7. The dependence vector for this code segment is $[1,-1]$ and is shown in Fig. 8.

If the code is transformed without considering the dependence vector, then we may write code for each space shown in Fig. 7 in any order. For example Fig. $6 \mathrm{~b}$ shows one such transformed code. This code is generated in the incorrect order [1, 5][1, 3], [1, 3][4, 5], [4, 5][4, 5] yielding errornous results when executed. To see why the ordering is incorrect, is illustrated by an example: $A[4][4]$ depends on $A[5][3]$ (write after read) and by generating code for the space $[1,5][1,3]$, we are violating this dependence. But considering the spaces using the order $[1,3][4,5],[4,5][4,5],[1,5][1,3]$ will generate correct code as shown in Fig. 6c. 
Fig. 7 Domain space

partitioning $(i>3 \& \& j>3)$
Fig. 8 Dependence vector for the example
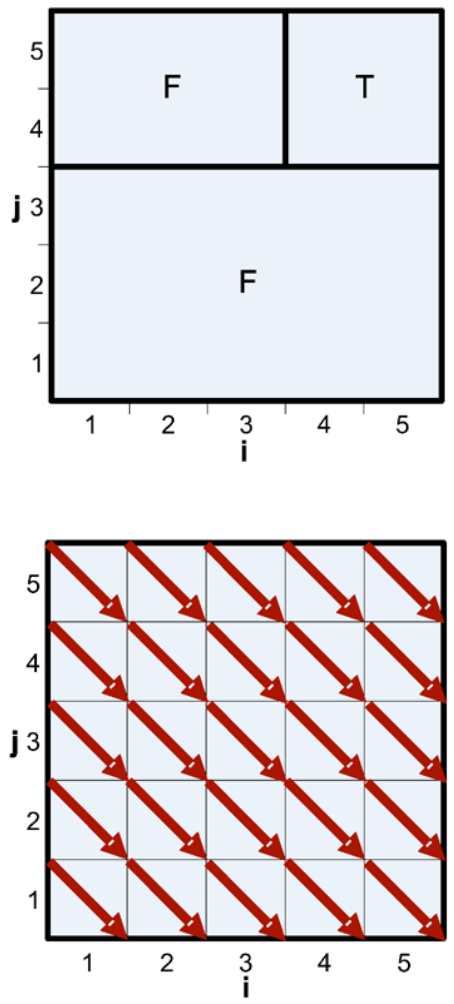

\subsection{Iteration space partitioning}

Recall that sets $T, F$ and $U$ were computed according to Sect. 4.1. Likewise, the dependence vector was computed in Sect. 4.2. We define the first problem of iteration space partitioning as below:

Problem 1 Given $T, F$ and $U$ and the dependence vector between the points in that space we are interested in $p=|T|+|F|+|U|$ sorted spaces $\left(S_{1}, S_{2}, \ldots, S_{p}\right)$ in a way that there is no loop-carried data dependence from $S_{i}$ to $S_{j}$ if $i<j$.

In general, solving Problem 1 requires finding the dependencies for the whole iteration space (i.e., solving equations $\forall k \in(1, \ldots, m) f_{k}\left(i_{1}, \ldots, i_{n}\right)=g_{k}\left(i_{1}, \ldots, i_{n}\right)$ in Fig. 5) for arbitrary equations, which is a known NP-hard [6] problem.

However, in two special cases, the problem can be solved efficiently. The first obvious case is when it is known (e.g., via a pragma directive) that there is no loop-carried data dependence. Here, the spaces can be sorted in any arbitrary way. The second case is when the dependency relationship is expressed as a linear equation of a special form. Specifically, if $f_{k}$ 's and $g_{k}$ 's in Fig. 5 can be expressed as:

$$
\begin{aligned}
& \forall k \in(1 . . n) f_{k}\left(i_{1}, i_{2}, \ldots, i_{n}\right)=f_{k}\left(i_{k}\right)=\alpha_{k, 1} \times i_{k}+\beta_{k, 1}, \\
& \forall k \in(1 . . n) g_{k}\left(i_{1}, i_{2}, \ldots, i_{n}\right)=g_{k}\left(i_{k}\right)=\alpha_{k, 2} \times i_{k}+\beta_{k, 2} .
\end{aligned}
$$


Fig. 9 Sort the spaces using the dependence vector

Fig. 10 Relational sort
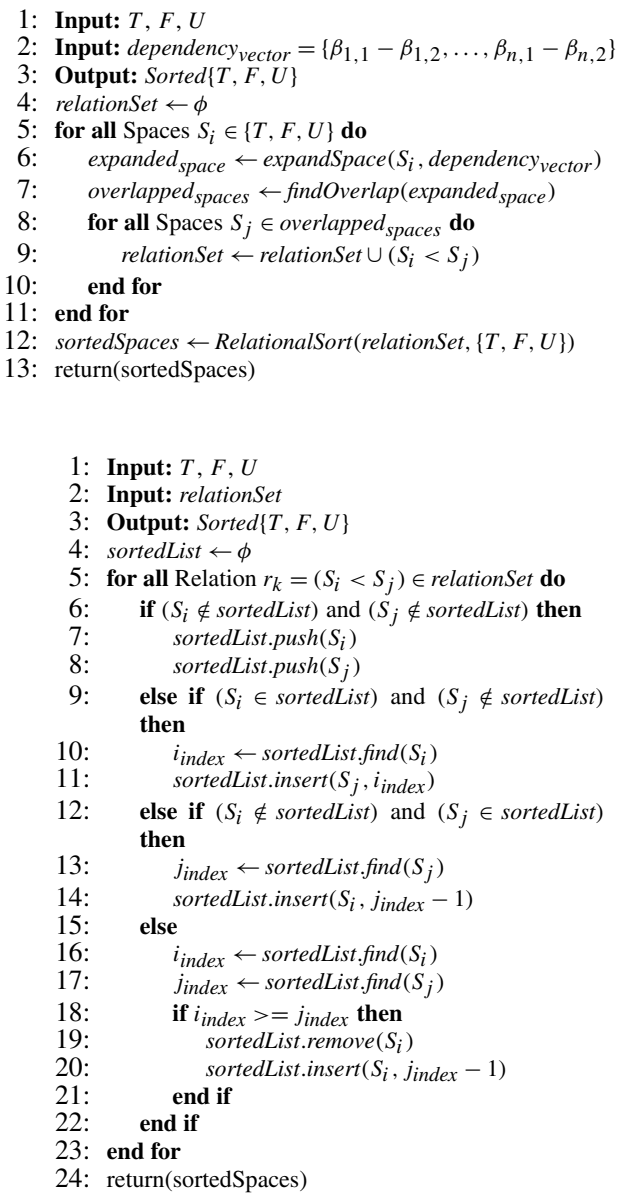

If $\forall k \alpha_{k, 1}=\alpha_{k, 2}$ then the dependence vector can be expressed as $\left\{\beta_{1,1}-\beta_{1,2}, \ldots\right.$, $\left.\beta_{n, 1}-\beta_{n, 2}\right\}$. Hence, Problem 1 can be re-defined as Problem 2 below:

Problem 2 Given $T, F$ and $U$ and the dependence vector in the form of $\left\{\beta_{1,1}-\right.$ $\left.\beta_{1,2}, \ldots, \beta_{n, 1}-\beta_{n, 2}\right\}$ we are interested in $p=|T|+|F|+|U|$ sorted spaces $\left(S_{1}, S_{2}, \ldots, S_{p}\right)$ in a way that there is no loop-carried data dependency from $S_{i}$ to $S_{j}$ if $i<j$.

Algorithm shown in Fig. 9 shows the proposed solution for Problem 2. This algorithm first expand the boundaries of all the spaces using the dependence vector (line 6). It then, finds all the spaces which have overlap with the expanded region, which gives, for each space, the set of dependent spaces (line 7). Using these dependencies, a set of relations between spaces is built (lines 8-10). Finally, algorithm in Fig. 10 is used as a subroutine to sort the spaces (line 12).

Algorithm shown in Fig. 10 works as follows. In a partially sorted list of spaces, if it reads a relation $S_{i}<S_{j}$ and if $S_{i}$ is located after $S_{j}$ in the list, their locations in the list are exchanged (lines 16-21). If any of $S_{i}$ and $S_{j}$ is not in the list, it is added to the list in a way to preserve the precedence relation (i.e. $S_{i}$ before $S_{j}$ if $S_{i}<S_{j}$ and etc.) (lines 6-15). 
Fig. 11 Example run of Algorithms 9 and 10

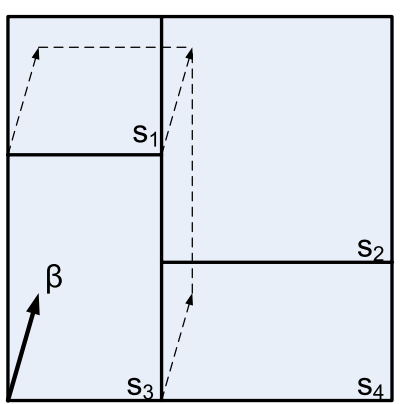

Relative set $=\left\{\left(\mathrm{s}_{3}<\mathrm{s}_{1}\right),\left(\mathrm{s}_{3}<\mathrm{s}_{2}\right)\right.$,
$\left.\left(\mathrm{s}_{3}<\mathrm{s}_{4}\right),\left(\mathrm{s}_{1}<\mathrm{s}_{2}\right),\left(\mathrm{s}_{4}<\mathrm{s}_{2}\right)\right\}$

(a)

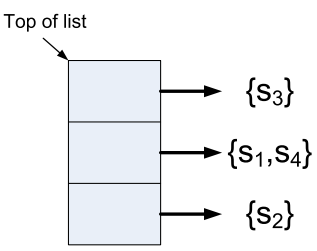

(b)$$
\text { i }
$$

\section{Sorted spaces $=$}

$\left\{\mathrm{s}_{3}, \mathrm{~s}_{1}, \mathrm{~s}_{4}, \mathrm{~s}_{2}\right\}$

(c)

Figure 11 shows an example run of algorithms in Figs. 9 and 10. Figure 11a shows the spaces that are dependent on the space $S_{3}$ by expanding the boundaries of $S_{3}$ using the dependence vector $\beta$. It also shows the relative set which is built by applying Algorithm 9 on all the spaces. Figure 11b shows the result of executing Algorithm 10 on the relative set shown in Fig. 11a and finally Fig. 11c shows the sorted spaces under the dependency vector $\beta$.

\subsection{Code generation}

Given the sorted spaces $\left(S_{1}, S_{2}, \ldots, S_{p}\right)$, code generation entails emitting a loop for the $S_{i}$ s. We note that, $S_{i}=\left[l_{1}, u_{1}\right] \times\left[l_{2}, u_{2}\right] \times \cdots \times\left[l_{n}, u_{n}\right]$. Hence, the loop control segment would be generated according to the following template:

$$
\begin{aligned}
& \text { for }\left(x_{1}=l_{1} ; x_{1} \leq u_{1} ; x_{1}++\right) \\
& \quad \text { for }\left(x_{2}=l_{2} ; x_{2} \leq u_{2} ; x_{2}++\right) \\
& \quad \vdots \\
& \quad \text { for }\left(x_{n}=l_{n} ; x_{n} \leq u_{n} ; x_{n}++\right) \\
& \quad \text { body }
\end{aligned}
$$

Moreover, the body of the generated loops contains only $s t_{\text {then }}$ if $S_{i} \in T$, only $s t_{\text {else }}$ if $S_{i} \in F$, or the original loop body if $S_{i} \in U$.

\section{Experiments}

To evaluate the proposed code transformation technique, several loop kernels from MediaBench [7] application suite and SPEC-2000 [10] were chosen. We also experimented with an $m p 3$ encoder implementation obtained from [1], an mpeg4 full motion estimation obtained from [2], GNU Image Manipulation Program (gimp) [14] and also qsdpcm [11] video compression algorithm which is obtained from [5].

By loop kernel, we mean the region of code that was impacted by the transformation. For example, if the transformed code was a conditional block within a for-loop, then the time taken to execute that entire for-loop before and after the optimization was used to determine the speedup. The characteristics of the loop kernels selected for our experiments are 
listed in Table 4. In Table 4 conditional expressions column shows the particular conditional expression(s). If there are more than one conditional expression in a loop kernel, then we run our algorithm for each instance of conditional expression separately (i.e., the algorithm is run iteratively as long as improvements are obtained). Also, in Table 4, Application column shows where we picked the loop kernel and Function description column shows the functionality of the code where the kernel is taken from. We applied our transformation technique (Sect. 4) at the source level to each of the chosen benchmarks by hand (except the first step in Sect. 4 which is automated), compiled the original and the transformed code, and measured the improvement. We did this experiment for four types of instruction sets: SPARC, x86, PowerPC and ARM. For all the instruction sets, we measured the speedup together with code size increase.

Each loop kernel (original and transformed) was compiled using different optimization levels of gcc [13], namely: no optimization (shown as no in the following sections); using $-O 1$ switch; using -O2 switch and finally using -O3 switch. In the following sections, the speedup calculations are based on the ratio of the time to execute the original loop kernel to the time to execute the optimized loop kernel. In each case the execution time before code transformation $\left(T_{o}\right)$ and the execution time after code transformation $\left(T_{n}\right)$ are measured and speedup has been calculated using the following formula: Speedup $=\left(T_{o} / T_{n}\right)$. Each bar in Figs. 12, 14, 16 and 18 shows the speedup after applying our code transformation. For each benchmark there are 5 bars, the first 4 representing the speedup for 4 cases of optimizations mentioned above, the fifth bar gives the average speedup. Likewise we have calculated the code size ratio, which is the transformed code size divided by the original code size.

\subsection{SPARC}

The results of experiments on SPARC are summarized in Table 5. The first half of Table 5 shows the result of measured time before and after transformation for 4 different optimization options. The second half of Table 5 shows the result of code size before and after transformation for the same 4 optimization options plus another optimization for code size $(-O s)$. The speedup and the code size ratio have been shown graphically in Figs. 12 and 13.

The experiments were run on a Sun workstation, with two $1503 \mathrm{MHz}$ SUNW, UltraSPARC-IIIi CPU's and 2 GB of memory, but the code ran for all experiments on a single CPU. We used GCC compiler version 3.4.1 in order to generate executables. In the best case, we observed application speedup of $6.58 \mathrm{X}$. On average, we observed application speedup of $2.34 \mathrm{X}$. On average we observed $2.51 \mathrm{X}$ increase on code size.

Note that there are cases where we measured decrease in code size (e.g., B9 or B15), this is due to removal of the conditional expression evaluation from the code combined with the small number of partitions that are generated. The same result is observed X86, PowerPC and ARM as shown in the following sections.

\subsection{Intel $\mathrm{x} 86$}

The results of experiments on Intel x 86 are summarized in Table 6. The first half of Table 6 shows the result of measured time before and after transformation for 4 different optimization options. The second half of Table 6 shows the result of code size before and after transformation for the same 4 optimization options plus another optimization for code size $(-O s)$. The speedup and the code size ratio have been shown graphically in Figs. 14 and 15.

The experiments were run on a MacBook with a Intel Dual Core $1.8 \mathrm{GHz}$ and $1 \mathrm{~GB}$ of memory. We used GCC compiler version 3.4.1 in order to generate executables. In the 


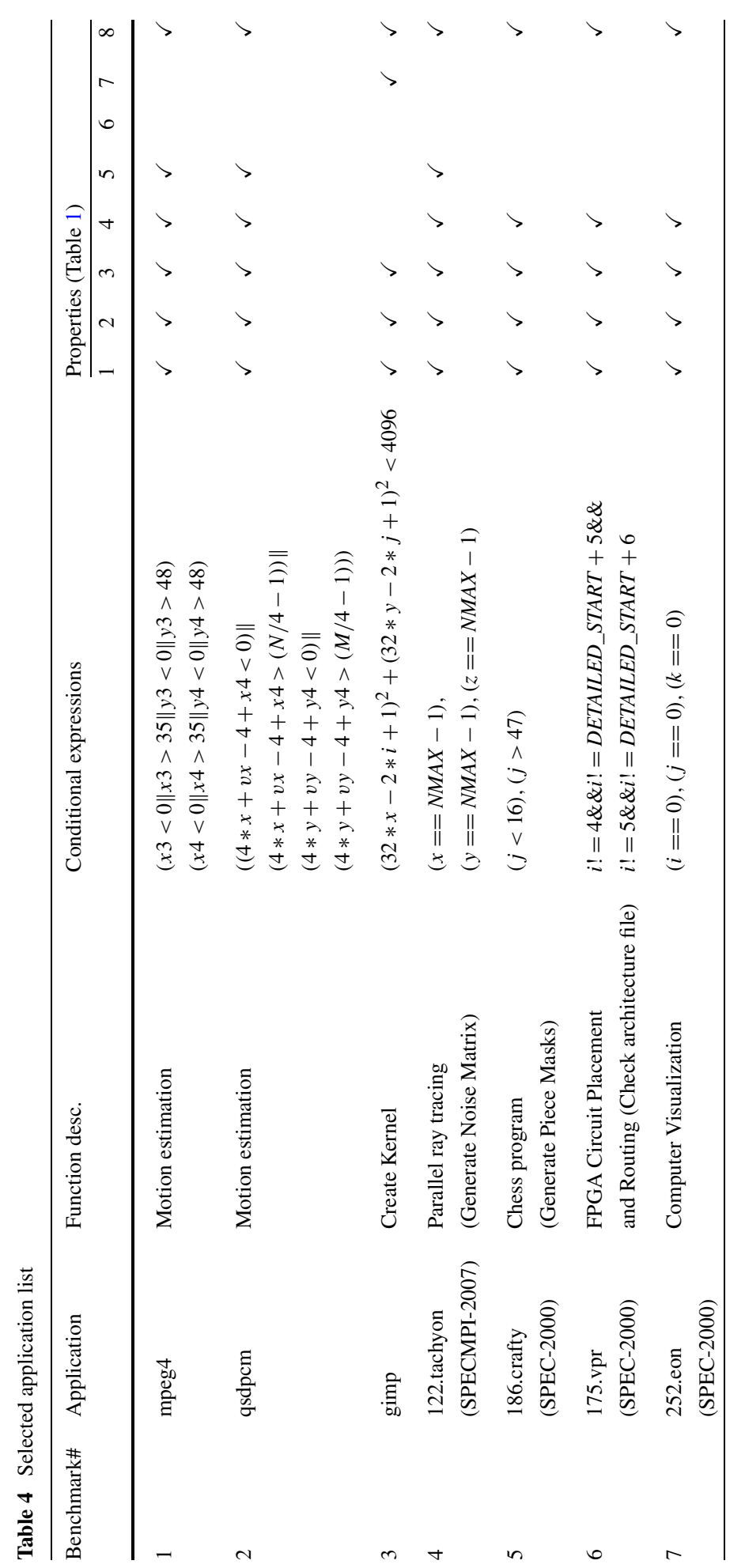




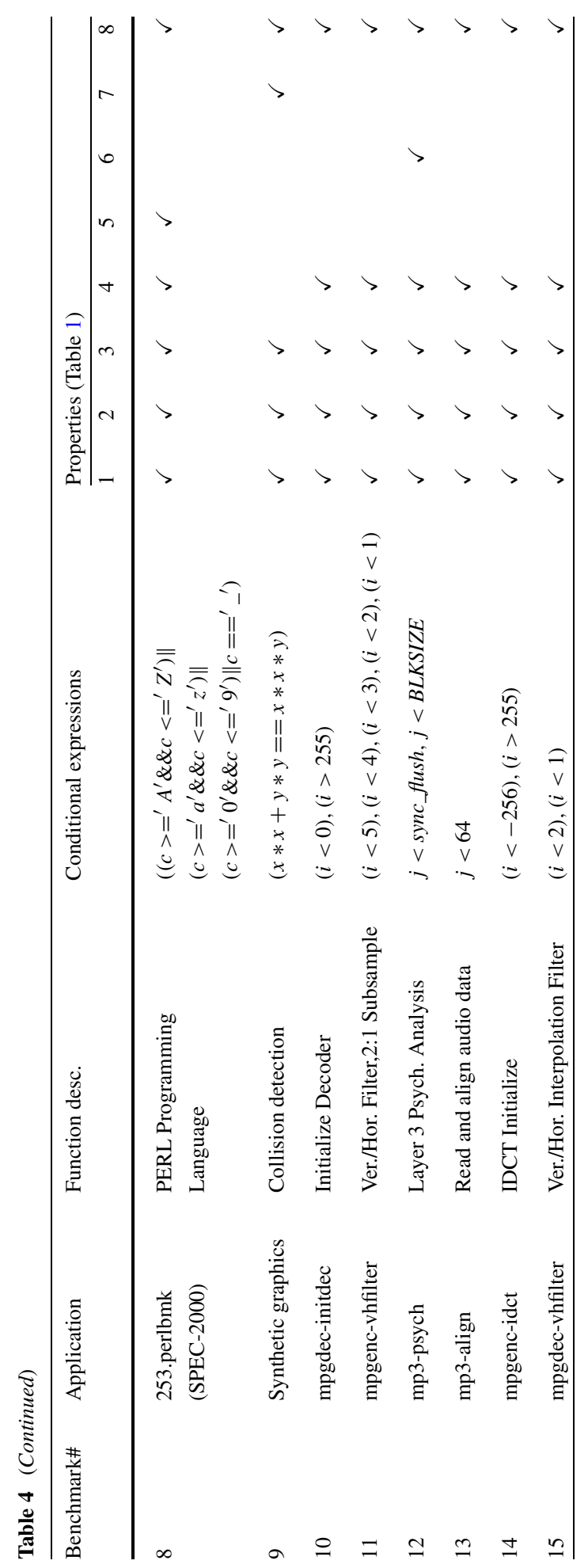




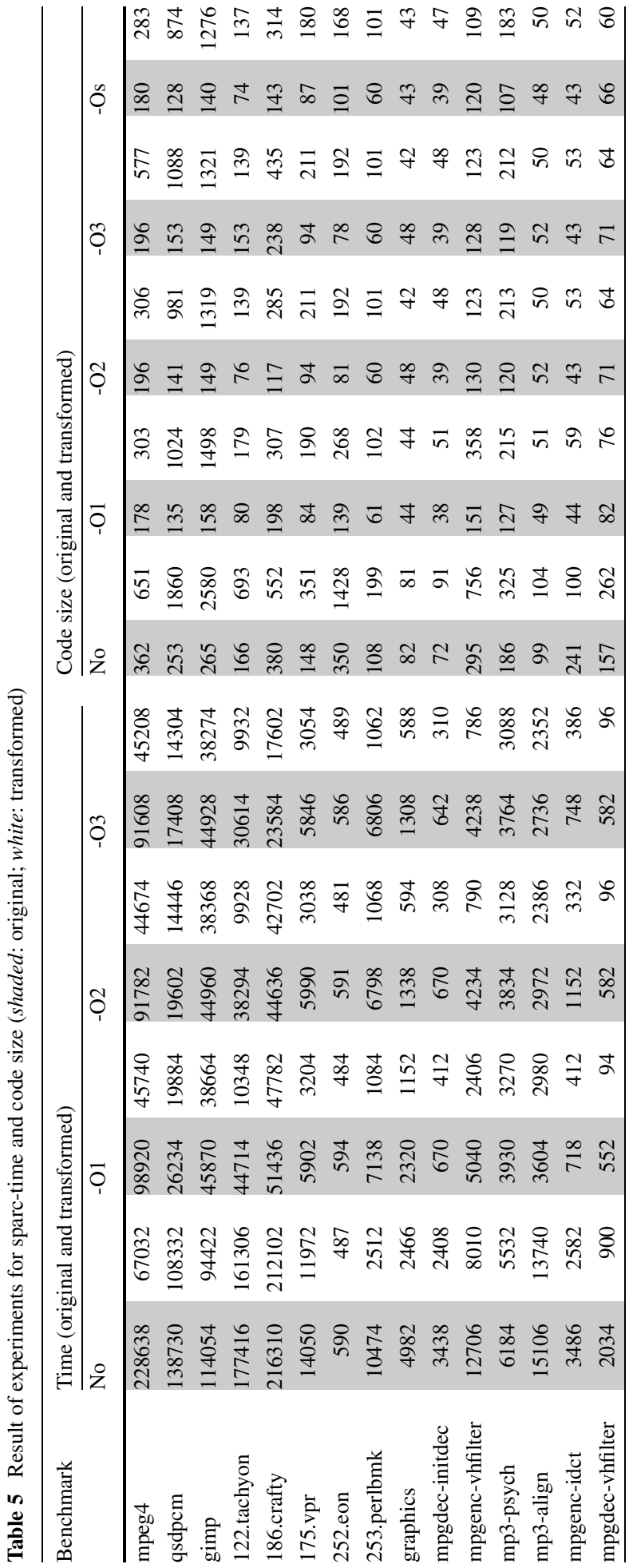




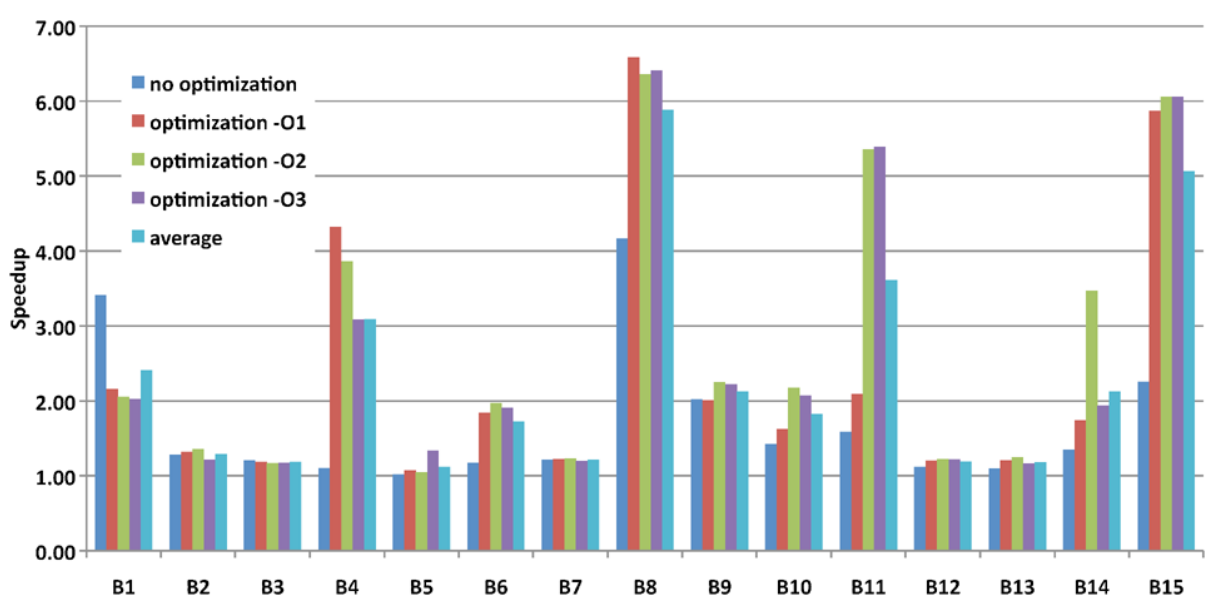

Fig. 12 Effect of transformation on time for SPARC

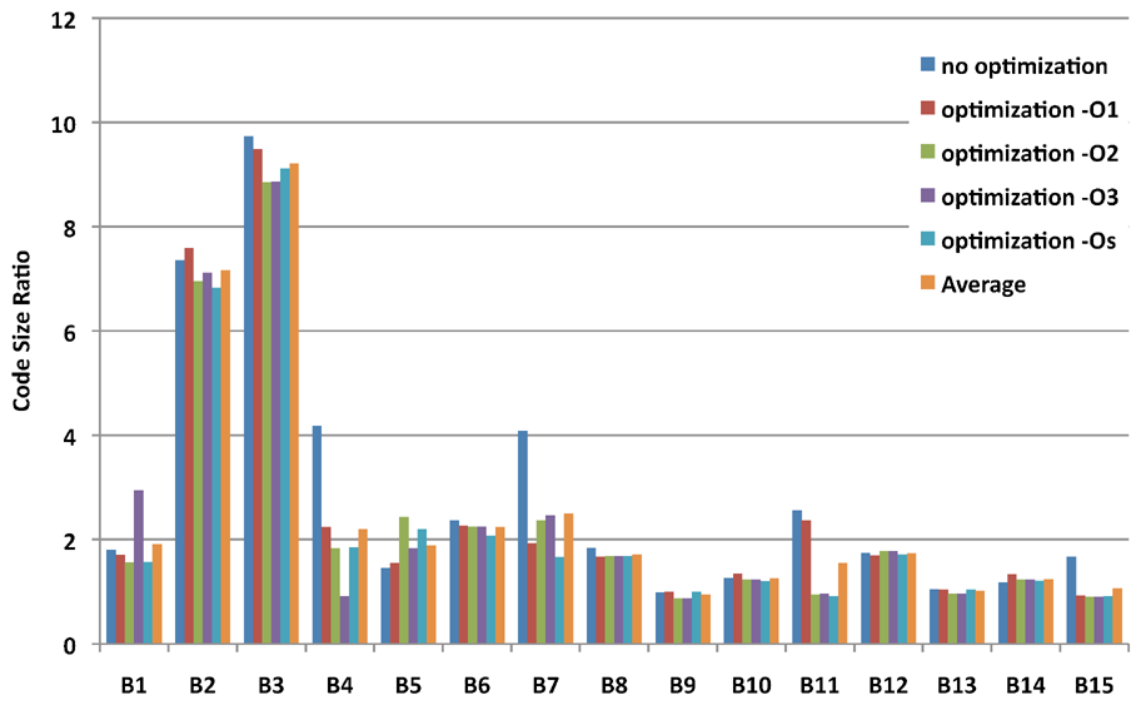

Fig. 13 Effect of transformation on code size for SPARC

best case, we observed application speedup of 10.65X. On average, we observed application speedup of 2.92X. On average we observed 2.34X increase on code size.

One interesting point here (and also for SPARC in Fig. 12) is the huge constant speedup for benchmark B4 with -O1, -O2 or -O3 optimization. In this benchmark there is an access to a 3 dimensional array. In the non-optimized version the address to the beginning of the array is always computed for every access inside loop. In the optimized version (-O1, -O2 and -O3), the beginning of the array computation is hoisted out of the loop and is kept in a register, yielding a huge performance gain. Finally, the timing result for all -O1, -O2 and -O3 is almost the same as can be seen in Tables 5 and 6 . 


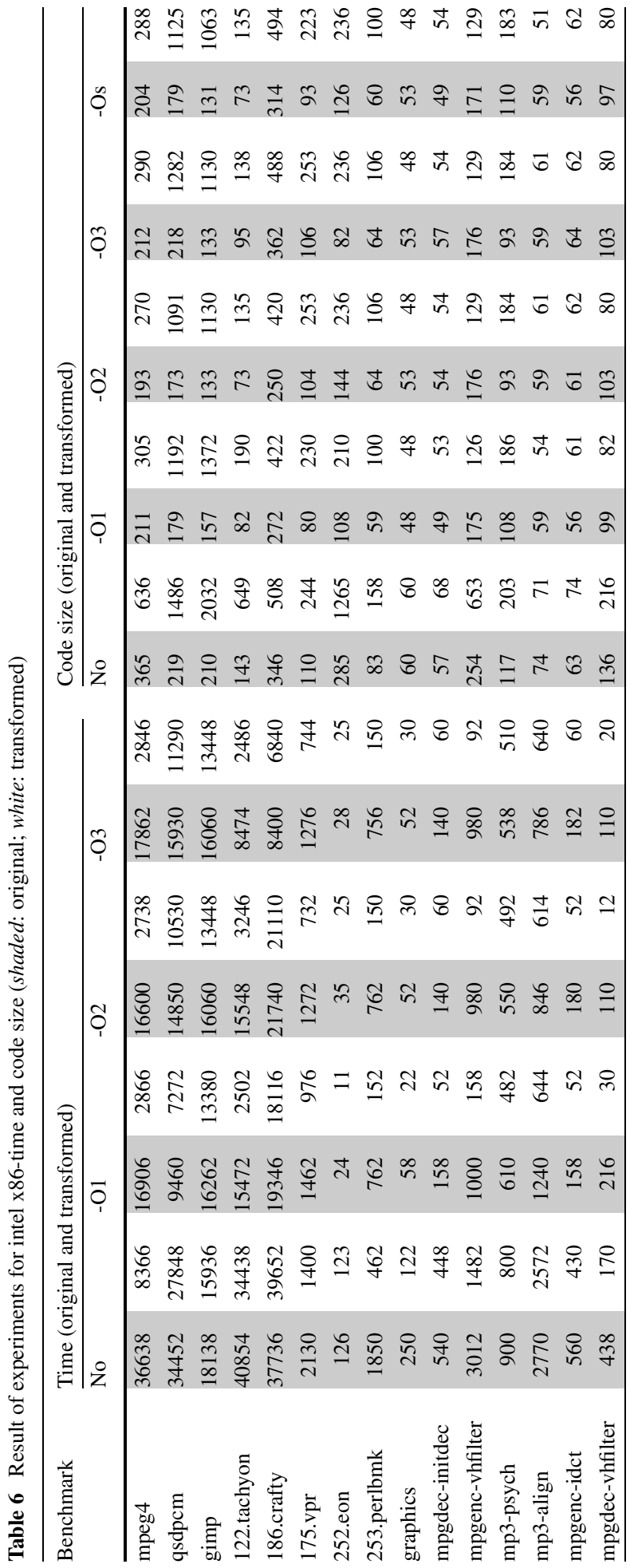




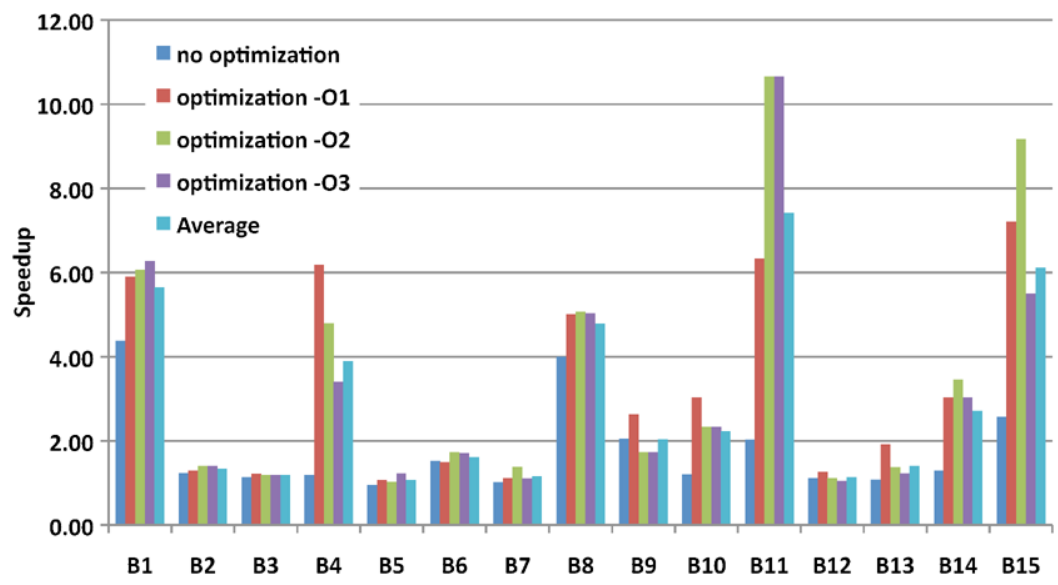

Fig. 14 Effect of transformation on time for $x 86$

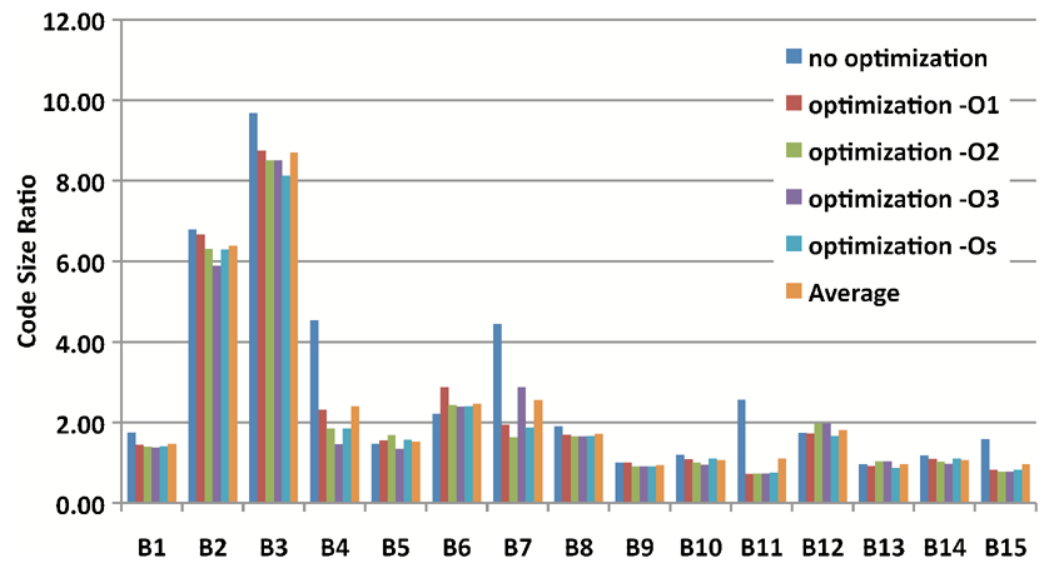

Fig. 15 Effect of transformation on code size for $x 86$

\subsection{PowerPC}

The results of experiments on PowerPC are summarized in Table 7. The first half of Table 7 shows the result of measured time before and after transformation for 4 different optimization options.

The second half of Table 7 shows the result of code size before and after transformation for the same 4 optimization options plus another optimization for code size $(-O s)$. The speedup and the code size ratio have been shown graphically in Figs. 16 and 17. The experiments were run on a Apple PowerMac G5 with a $1.6 \mathrm{GHz}$ PowerPC G5 and $768 \mathrm{MB}$ of memory. We used GCC compiler version 4.0.1 in order to generate executables. In the best case, we observed application speedup of 9.33X. On average, we observed application speedup of 2.44X. On average we observed $2.31 \mathrm{X}$ increase on code size. 


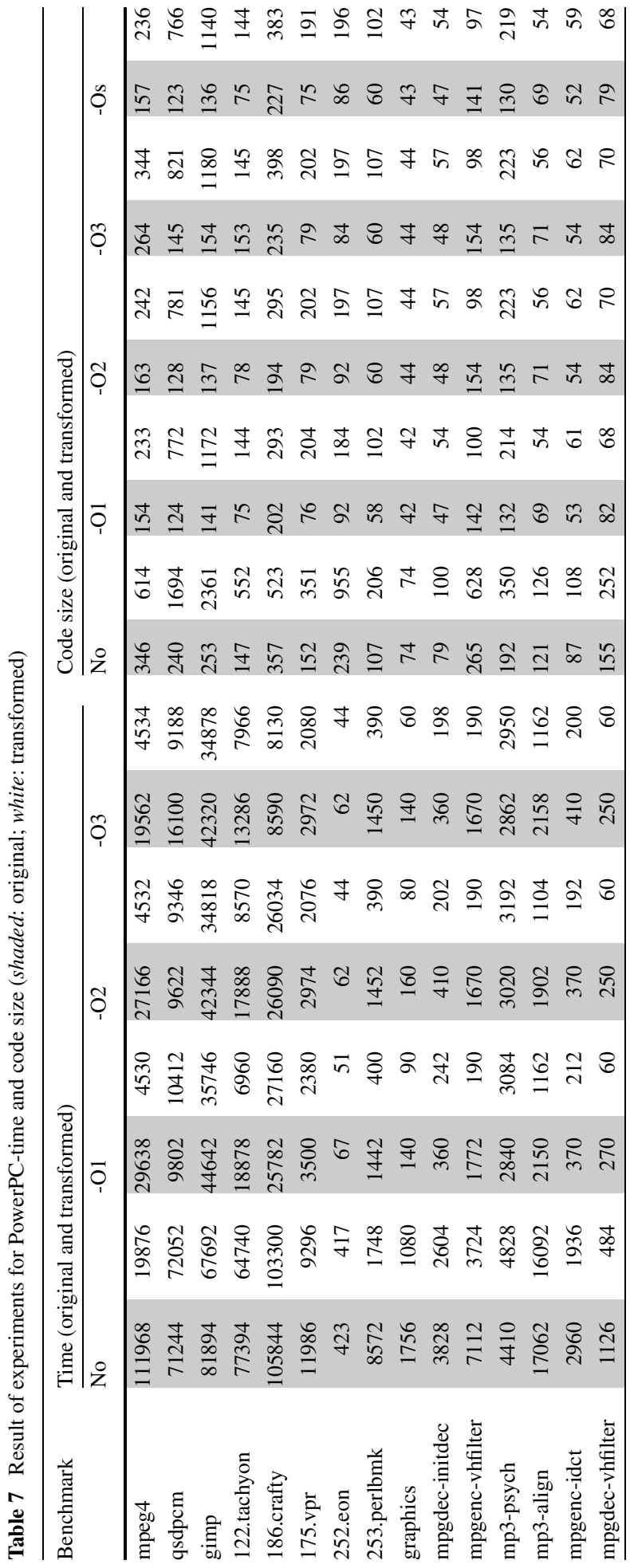




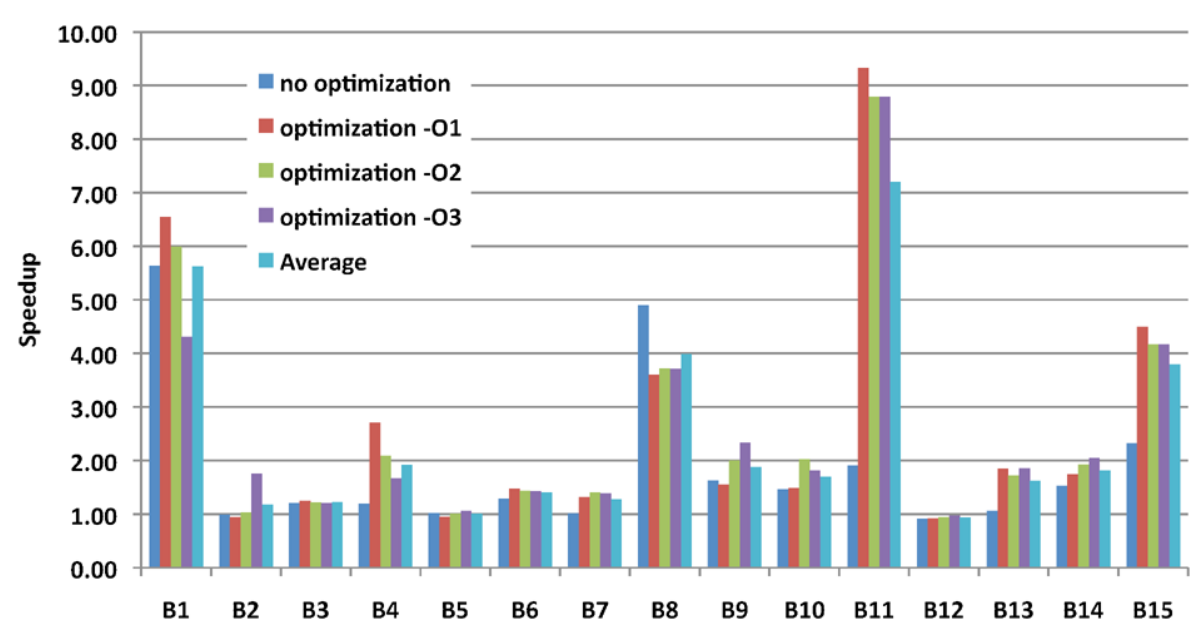

Fig. 16 Effect of transformation on time for PowerPC

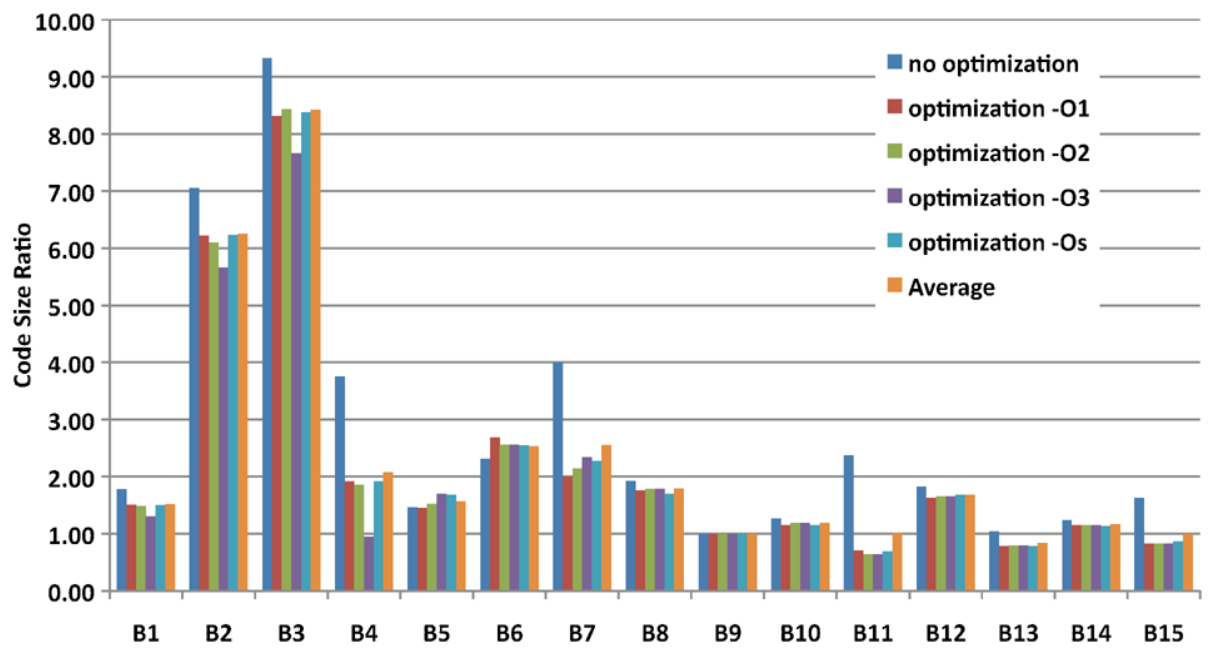

Fig. 17 Effect of transformation on code size for PowerPC

\subsection{ARM}

The results of experiments on ARM are summarized in Table 8. The first half of Table 8 shows the result of measured time before and after transformation for 4 different optimization options. The second half of Table 8 shows the result of code size before and after transformation for the same 4 optimization options plus another optimization for code size $(-O s)$. The speedup and the code size ratio have been shown graphically in Figs. 18 and 19.

The experiments were run on an ARM evaluation board TS-7250 from Technologic Systems [12] with a $200 \mathrm{MHz}$ ARM9 which its characteristics are shown in Table 9. We used arm-linux-gcc compiler version 3.4.4 in order to generate executables. In the best case, we observed application speedup of 5.77X. On average, we observed application speedup of 2.04X. On average we observed 3.23X increase on code size. 


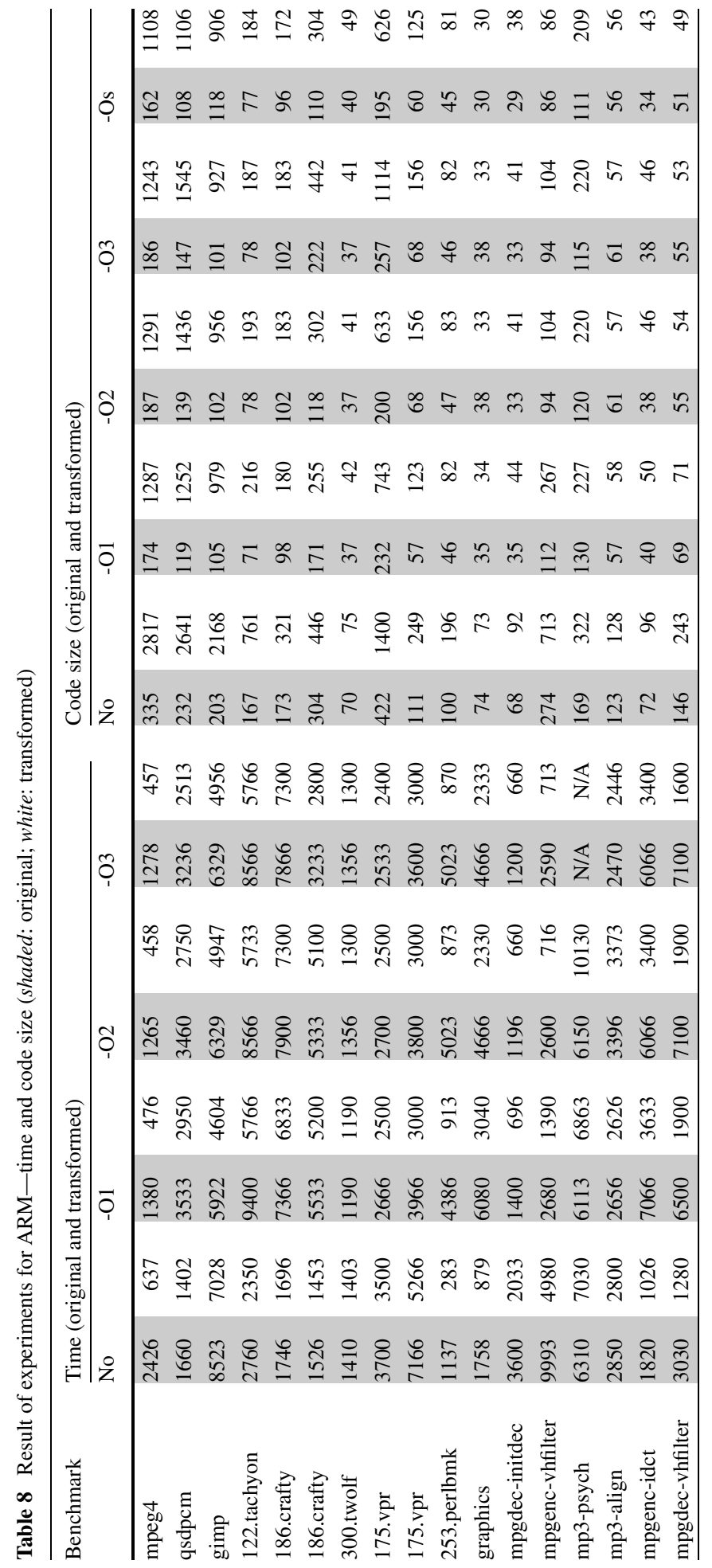




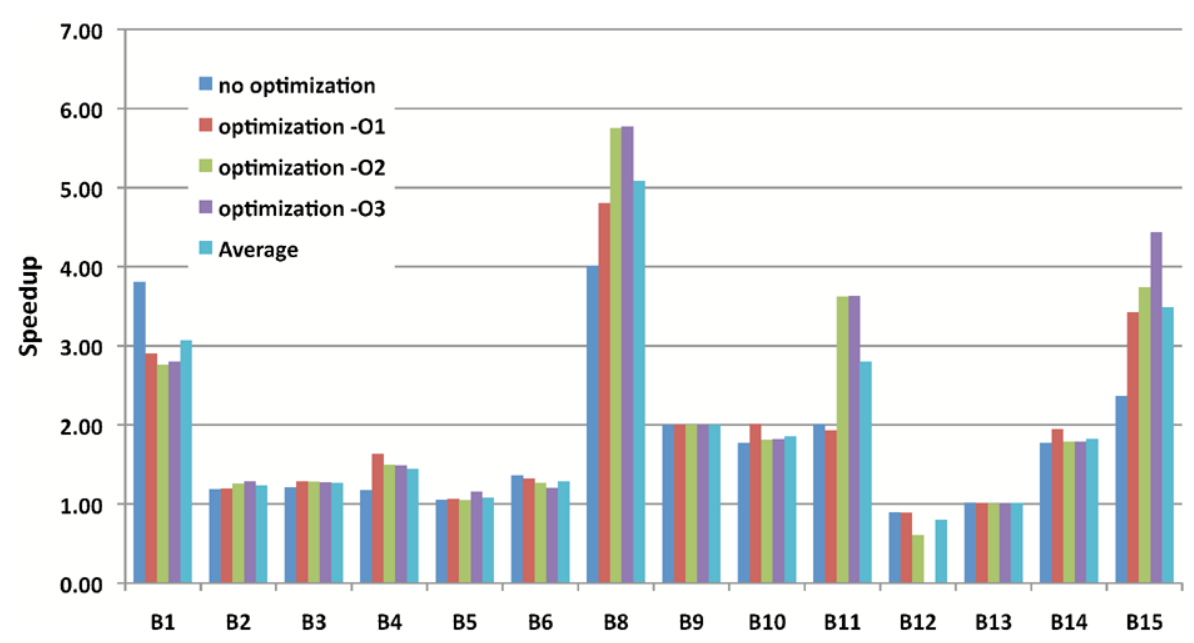

Fig. 18 Effect of transformation on time for ARM

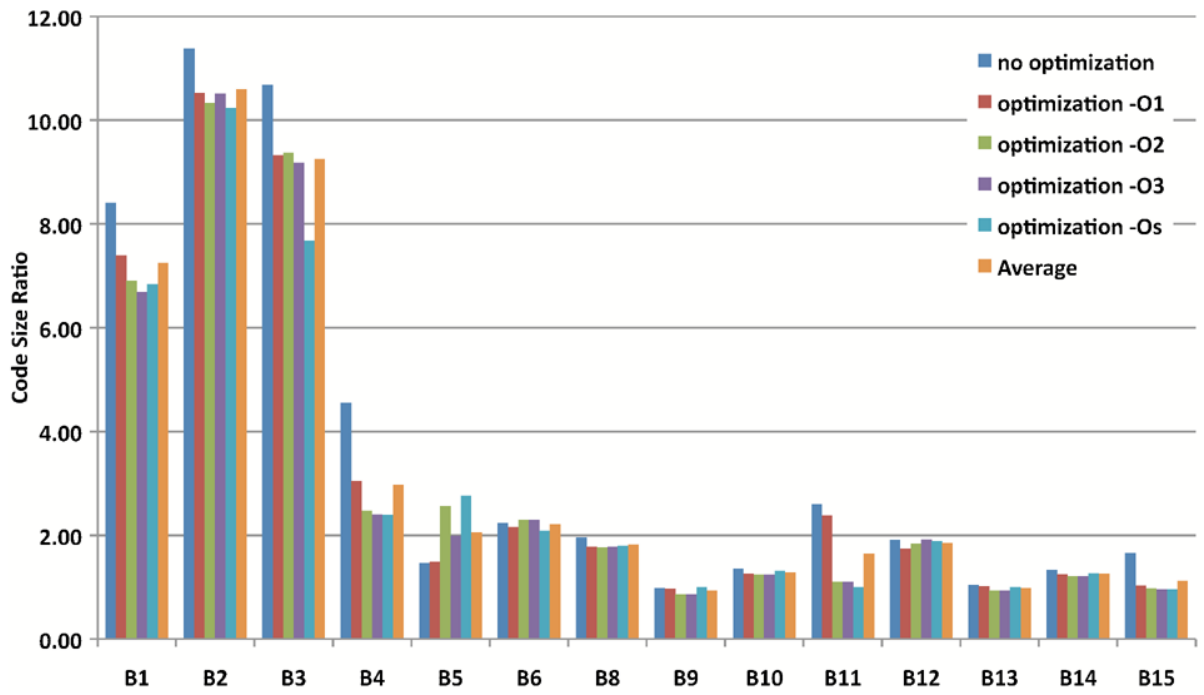

Fig. 19 Effect of transformation on code size for ARM

This technique is not always rewarding as can be seen from the result for B12 on ARM9 processor (Fig. 18). This is due to high number of memory array accesses inside the original loop. In the original loop, each array access is loaded once through out the loop. When partitioned in a series of loops (after transformation), each loop partition will have its own memory loads, which makes it more expensive compared to the original code.

\subsection{Full application speed-up}

To investigate the benefit of our proposed transformation on a whole application, we tested our transformation on 3 applications, namely mpeg decoder, mpeg encoder and qsdpcm on 
Table 9 ARM9 board specification

Fig. 20 Full application performance improvement

Fig. 21 Full application code size increase
$200 \mathrm{MHz}$ ARM9 processor with MMU

$32 \mathrm{MB}$ of High Speed SDRAM

32 MB Flash disk used for RedBoot boot-loader,

Linux kernel and root file system

Linux Kernel 2.6.20

USB Flash drive supported

10/100 Ethernet interface

2 USB 2.0 Compatible OHCI ports (12 Mbit/s Max)
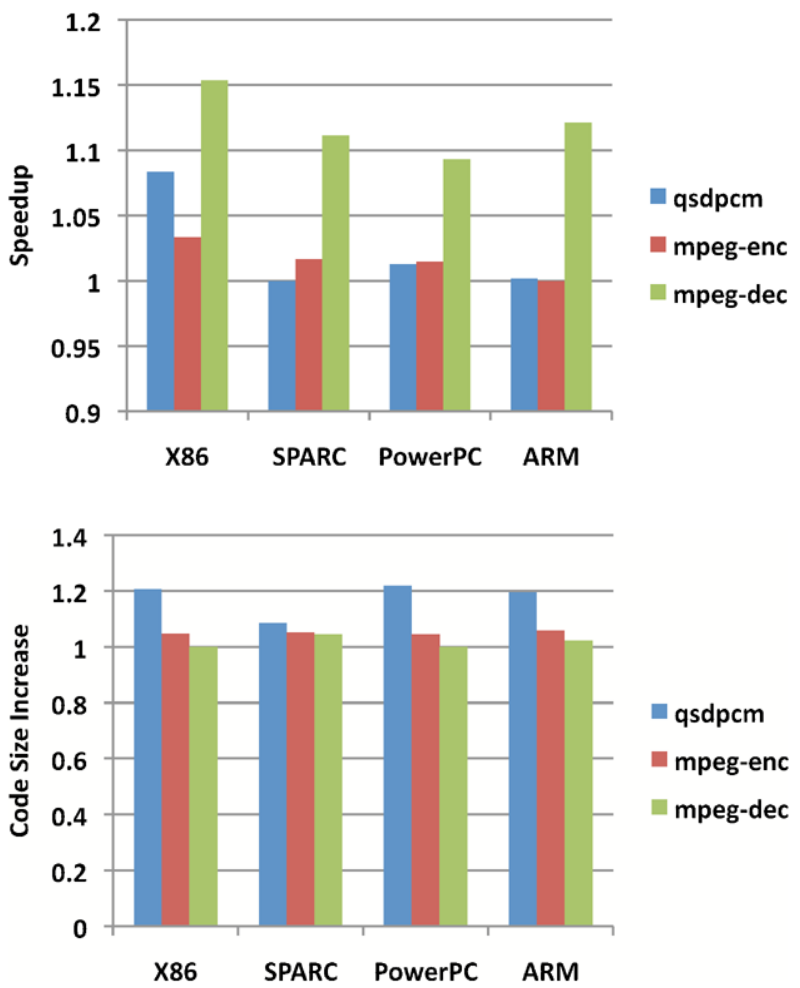

all the 4 previously mentioned instruction sets: SPARC, $x 86$, PowerPC and ARM. For this experiment we only used $g c c-O 3$ for compilation and as before we hand transformed all the places in code which our transformation could be applied. Figure 20 shows the result of the performance improvement for the three mentioned application. In the best case we get $15 \%$ speedup for the mpeg-dec on X86. On average we get 5\% performance improvement. Figure 21 shows the result of the code size increase for the three mentioned application. In the worst case we get $21 \%$ code size increase for the $q s d p c m$ on PowerPC. On average we get $6 \%$ code size increase.

It's worth mentioning that like any other compiler optimization (e.g. most of the compiler optimization techniques mentioned in Table 4), our technique is applicable to certain part of code, which may or may not be part of the hot spots. So, the gain that we can get on the full application speedup varies a lot. Even though, there are compiler optimization techniques 
that by enabling some hardware techniques can gain a lot (like all the loop parallelization techniques), no single software-only compiler optimization can give us huge speed-ups on all the benchmarks, when taking the entire application into account.

\subsection{Additional remarks}

1. Experiments with GCCs increasing levels of optimizations (none, -O1, -O2, -O3) show that the proposed optimization techniques yields additional performance improvements when applied in conjunction with existing compiler optimizations in vast majority of cases. This is due to dataflow optimizations which will be enabled because of the removal of the conditional expressions from loop bodies. In the few cases where this is not true (e.g., 186.crafty in Intel or PowerPC or qsdpcm in PowerPC), the difference is within measurement noise. Or sometimes, it is because more optimization (i.e. -O2, -O3) will not give more benefits comparing to -O1. This was shown for example in 122.tachyon in both Figs. 12 and 14.

Furthermore, this is a well known effect of interactions between compiler optimizations and is indeed also visible without our transformations (e.g., 175.vpr for SPARC and qsdpcm for Intel x86 and PowerPC) as shown in Tables 5, 6 and 7.

2. Note that since there are real runtime results on real machines, they naturally factor in any possible performance effects of code size increase on caching. Thus the speedups are the real effect of the transformation on actual running code.

3. The code size increase reported in Sects. 5.1, 5.2, 5.3 and 5.4 are only for the loop kernels.

4. The domain space partitioning algorithm might produce lots of spaces for a given nested conditional expression. If the loop is partitioned based on all these spaces, then the loops overhead will create a diminish return for performance gain. A similar argument has been presented in [4]. What should be done in these cases is to drop all those spaces which have a size smaller than a constant threshold, in this way they will be merged with unknown spaces and the original code will be generated for them in the code generation step.

5. Given profiling information, our method can be applied to a general loop that has variable lower and upper bounds: (1) Separate out the part of domain space with the profiled upper/lower bounds, (2) apply the method presented in this paper and generate optimized code for this carved out space, and finally (3) for the remaining part of the domain space, use the original code. As an example, consider the loop shown here:

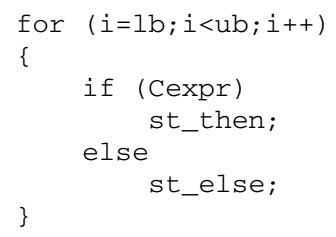

If profiling information shows that there is an interval $[L p, U p]$ ( $L p$ and $U p$ are two constant integers) such that $l b \leq L p \leq U p \leq u b$, we can transform the code as follows:

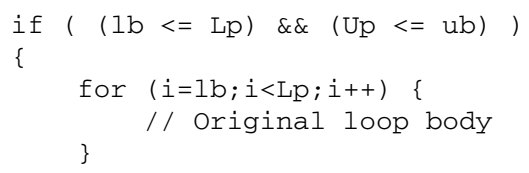




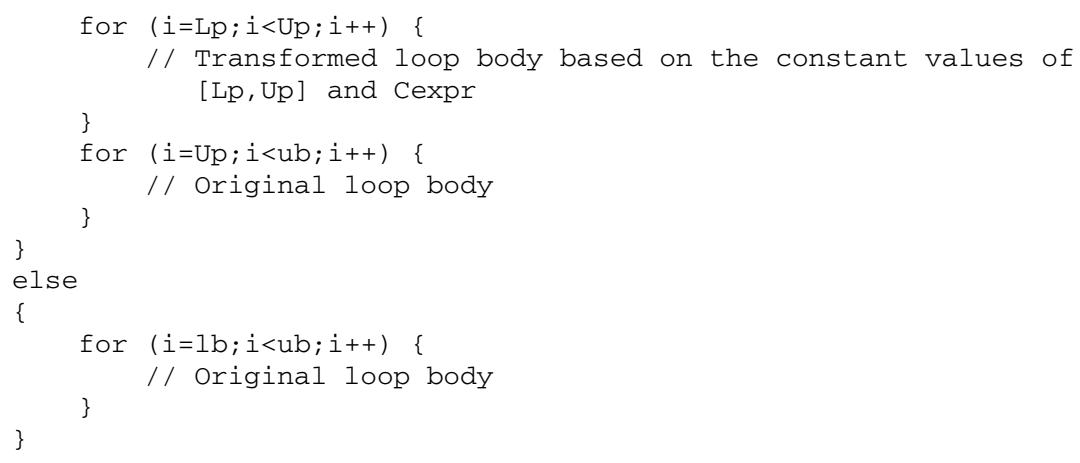

\section{Conclusion}

Given the stringent design constraints, performance requirements of embedded systems and as software is becoming a larger fraction of engineering effort, the importance of aggressive compiler optimizations also increases. Hence, it is acceptable for a compiler intended for embedded computing to take longer to execute but perform aggressive compiler optimizations. We have presented a new loop transformation technique, intended for embedded compilers. The transformation technique optimizes loops with nested conditional blocks and it decomposes the loop nests in a way that conditional testing is eliminated. Applying the proposed transformation technique on the loop kernels taken from Mediabench, SPEC2000, mpeg4, qsdpcm and gimp, on average we measured a 2.34X speedup when running on a UltraSPARC processor, a $2.92 \mathrm{X}$ speedup when running on an Intel Core Duo processor, a 2.44X speedup when running on a PowerPC G5 processor and a 2.04X speedup when running on an ARM9 processor. In addition to ARM9 which is a representative of an embedded processor, we used high-end processors because better compilers are available, so as to avoid the possibility that our technique looks better than it should because of poor optimizations done by the compiler. Also, these processors are representative of high-end embedded processors (Intel Core Duo has an embedded version, so do PowerPC and SPARC). On average, we measured a code size increase of 2.51X for SPARC, 2.34X for Intel x86, 2.31X for PowerPC and 3.23X for ARM. Note that despite the size increase, the overall performance is still improved by the above factors, i.e., cache performance degradation, if any, due to the increased code size is already factored into the results, since we measured actual runtime of the original and transformed code. Performance improvement, taking the entire application into account, was also promising: for 3 selected applications (mpeg-enc, mpeg-dec and $q s d p c m$ ) we measured $15 \%$ speedup on best case (5\% on average) for the whole application.

Acknowledgements This work was in part supported by grant \#0749508 from the National Science Foundation.

Open Access This article is distributed under the terms of the Creative Commons Attribution Noncommercial License which permits any noncommercial use, distribution, and reproduction in any medium, provided the original author(s) and source are credited.

\section{References}

1. Iso mp3 sources. Available as http://www.mp3-tech.org/programmer/sources/dist10.tgz

2. Falk H, Marwedel P (2003) Control flow driven splitting of loop nests at the source code level. In: Proceedings of DATE, pp 410-415 
3. Ghodrat MA, Givargis T, Nicolau A (2005) Equivalence checking of arithmetic expressions using fast evaluation. In: Proceedings of the CASES, pp 147-156

4. Ghodrat MA, Givargis T, Nicolau A (2007) Short-circuit compiler transformation: Optimizing conditional blocks. In: Proceedings of the 12th Asia and South Pacific design automation conference (ASPDAC 2007), pp 504-510

5. Issenin I, Dutt N (2006) Data reuse driven energy-aware mpsoc co-synthesis of memory and communication architecture for streaming applications. In: CODES-ISSS 2006, pp 294-299

6. Kennedy K, Allen R (2001) Optimizing compilers for modern architectures: A dependence-based approach. Morgan Kaufmann, San Mateo

7. Lee $\mathrm{C}$ et al (1997) Mediabench: A tool for evaluating and synthesizing multimedia and communications systems. In: International symposium on microarchitecture, pp 330-335

8. Moore RE (1966) Interval analysis. Englewood Cliffs, Prentice-Hall

9. Muchnick SS (1997) Advanced compiler design and implementation. Morgan Kaufmann, San Mateo

10. Standard Performance Evaluation Corporation Spec cpu2000. Available as http://www.spec.org/ cpu2000/

11. Stobach P (1988) A new technique in scene adaptive coding. In: Proceedings of EUSIPCO

12. Technologic systems http://www.embeddedarm.com/products/board-detail.php?product=TS-7250

13. The GCC Team. Gnu compiler collection. Available as http://gcc.gnu.org/

14. The GIMP Team. Gnu image manipulation program. Available as http://www.gimp.org/

15. Wolfe M (1995) High-performance compilers for parallel computing. Addison-Wesley, Reading

16. Wolfe M (2005) How compilers and tools differ for embedded systems. In: Proceedings of the CASES, p 1 